\title{
Vitamin D in relation to incident sarcopenia and changes in muscle parameters among older adults: the KORA-Age study
}

\author{
Romy Conzade $^{1}$ Eva Grill ${ }^{2} \cdot$ Heike A. Bischoff-Ferrari ${ }^{3} \bullet$ Uta Ferrari $^{4} \cdot$ Alexander Horsch $^{5} \bullet$ Wolfgang $^{-}$ \\ Koenig $^{6,7,8} \cdot$ Annette Peters $^{1} \cdot$ Barbara Thorand $^{1, 凶}$ \\ ${ }^{1}$ Helmholtz Zentrum München, German Research Center for Environmental Health (GmbH), Institute of \\ Epidemiology, 85764 Neuherberg, Germany \\ ${ }^{2}$ Institute for Medical Information Processing, Biometrics and Epidemiology, Ludwig Maximilian University of \\ Munich, 81377 Munich, Germany \\ ${ }^{3}$ Centre on Aging and Mobility, University of Zurich and City Hospital Waid, 8091 Zurich, Switzerland \\ ${ }^{4}$ Department of Medicine IV, University Hospital, Ludwig Maximilian University of Munich, 80336 Munich, \\ Germany \\ ${ }^{5}$ Department of Computer Science, UiT - The Arctic University of Norway, 9037 Troms $\varnothing$, Norway \\ ${ }^{6}$ Deutsches Herzzentrum München, Technische Universität München, 80636 Munich, Germany \\ ${ }^{7}$ DZHK (German Centre for Cardiovascular Research), Partner Site Munich Heart Alliance, 80802 Munich, \\ Germany \\ ${ }^{8}$ Institute of Epidemiology and Biostatistics, University of Ulm, 89081 Ulm, Germany
}

$\triangle$ B. Thorand, thorand@helmholtz-muenchen.de, +49(0) $893187-4480$

\section{ORCID ID}

Romy Conzade: 0000-0002-5667-6849; Eva Grill: 0000-0002-0273-7984; Heike A. Bischoff-Ferrari: 00000003-0264-6318; Uta Ferrari: 0000-0002-8354-5721; Alexander Horsch: 0000-0001-7745-0139; Wolfgang Koenig: -; Annette Peters: 0000-0001-6645-0985; Barbara Thorand: 0000-0002-8416-6440

Acknowledgments We thank all participants of the KORA-Age study for their extraordinary commitment and goodwill. The KORA study was initiated and financed by the Helmholtz Zentrum München - German Research Center for Environmental Health, which is funded by the German Federal Ministry of Education and Research (BMBF) and by the State of Bavaria. The KORA-Age project was financed by the German Federal Ministry of Education and Research (BMBF FKZ 01ET0713 and 01ET1003A) as part of the 'Health in old age' program. RC is supported by a $\mathrm{PhD}$ scholarship from the 'Studienstiftung des deutschen Volkes'. The funders had no role in study design, data collection and analysis, decision to publish, or preparation of the manuscript.

Authors' contribution RC and BT conceived and designed the study. RC analyzed the data and wrote the first draft of the paper. EV, HBF and BT, as members of the PhD Thesis Committee of RC, helped in interpreting the data. All authors revised the paper critically for intellectual content and approved the final version. All authors agree to be accountable for the work and to ensure that any questions relating to the accuracy and integrity of the paper are investigated and properly resolved.

\section{Compliance with Ethical Standards}

Conflicts of interest Romy Conzade, Eva Grill, Heike A. Bischoff-Ferrari, Uta Ferrari, Alexander Horsch, Wolfgang Koenig, Annette Peters and Barbara Thorand declare that they have no conflict of interest.

Ethical Approval, Human Rights and Informed Consent The KORA-Age was approved by the Ethics Committee of the Bavarian Medical Association (reference number 08064). Written informed consent has been obtained from the participants and all investigations have been conducted according to the principles expressed in the Helsinki declaration. 


\begin{abstract}
Summary We report low baseline 25-hydroxyvitamin D (25OHD) levels being associated with unfavorable changes in muscle mass and physical performance over three years, but not with incident sarcopenia. Future prospective studies are needed to assess causality and to address the issue of competing risks such as mortality in older cohorts.

Introduction Effects of low serum 25-hydroxyvitamin D (25OHD) on age-related changes in muscle mass and function remain unclear. Our aims were to explore associations of baseline 25OHD levels with prevalent and incident sarcopenia and changes in muscle parameters, and to examine the role of parathyroid hormone (PTH) therein.

Methods Cross-sectional ( $n=975)$ and prospective analyses $(n=702)$ of older adults aged 65-93 years participating in the KORA-Age study. Sarcopenia was defined using the 2010 European Working Group on Sarcopenia in Older People (EWGSOP) criteria as low muscle mass combined with low grip strength or low physical performance. Associations with baseline 25OHD were examined in multiple regression analyses.

Results Low vitamin D status was linked to increased odds of prevalent sarcopenia. Over three years, low baseline $25 \mathrm{OHD}<25$ vs. $\geq 50 \mathrm{nmol} / \mathrm{L}$ were associated with greater loss of muscle mass and increased time for the Timed Up and Go test. The risk for developing incident sarcopenia was not significantly elevated in individuals with low baseline 25OHD but when including death as combined outcome alongside incident sarcopenia, there was a strong positive association in multivariable analysis (OR (95\% CI): 3.19 (1.54-6.57) for $25 \mathrm{OHD}<25 v s$. $\geq 50 \mathrm{nmol} / \mathrm{L}$ ). There was no evidence for a PTH-mediating effect.

Conclusion Low baseline 25OHD levels were associated with unfavorable changes in muscle mass and physical performance, but not with incident sarcopenia. Future randomized trials are needed to assess causality and to address the issue of competing risks such as mortality in older cohorts.
\end{abstract}

Keywords Vitamin D $\bullet$ Sarcopenia $\bullet$ Muscle changes $\bullet$ Prospective $\bullet$ Older adults

Electronic Supplementary material The version of this article contains electronic supplementary material. 


\section{Introduction}

Conceptualized as a geriatric syndrome of the gradual loss of muscle mass and function, sarcopenia becomes increasingly common in older age and often occurs simultaneously with low serum 25-hydroxyvitamin D (25OHD) levels [1]. Both conditions are linked to common clinical geriatric outcomes, including muscle weakness, falls and frailty [2].

There are several reasons to consider how vitamin D may have a beneficial effect on muscle health, yet individual observational studies examining the relationship of $25 \mathrm{OHD}$ levels with sarcopenia and related muscle parameters have yielded inconsistent results and the number of prospective studies is limited. We are aware of only two prospective studies that have examined 25OHD in relation to the incidence of sarcopenia defined by both low muscle mass and low muscle function. While the first study did not observed a significant association between vitamin D status and incident sarcopenia [3], low levels of 25OHD were associated with a significant increased risk of incident sarcopenia in the second one [4]. Furthermore, low 25OHD levels were not linked to change in muscle mass over time $[3,5,6]$. In some prospective studies, lower 25OHD levels were associated with loss of muscle function such a muscle strength or physical performance [5-8], while others did not find respective associations [3,9-11].

The mechanisms by which vitamin D status may affect muscle metabolism and function are not fully elucidated [12]. They may be both direct, via activation of a vitamin D receptor on muscle tissue by the biologically active form of vitamin D [13], and indirect, via effects of low serum calcium [14], low serum phosphate [15] or increased serum parathyroid hormone (PTH) levels [6].

We used cross-sectional and prospective data from the population-based KORA (Cooperative Health Research in the Region of Augsburg)-Age study to determine the associations of baseline 25OHD levels with prevalent and incident sarcopenia as well as changes in related muscle parameters in German older adults. The role of PTH as a potential mediator was also examined.

\section{Methods}

\section{Study design and participants}

We used data from two time points of the KORA-Age study (baseline: 2008/2009; follow-up: 2012). Full details about the study design and participants have been published previously [16]. Briefly, the cohort includes 1079 eligible participants aged $\geq 65$ years on 31.12.2008, who participated in a multidimensional health assessment at baseline. A total of 975 individuals with complete health assessment and without hypercalcemia at baseline (serum calcium levels $>2.6 \mathrm{mmol} / \mathrm{L}$ ) were included in cross-sectional analyses. After exclusion of individuals with sarcopenia at baseline, without follow-up data or with missing information on sarcopenia at follow-up, a total of 702 participants were included in prospective analyses. Of those without follow-up data $(n=179), 57$ died and 122 did not participate due to other reasons. The Ethics Committee of the Bavarian Medical Association approved the study protocol (reference number: 08064), and written informed consent was obtained from all participants.

\section{Sarcopenia and related muscle parameters}

Sarcopenia status was assessed at baseline and follow-up using the European Working Group on Sarcopenia in Older People (EWGSOP) 2010 criteria: low muscle mass combined with either low muscle strength or low physical performance [17].

Muscle mass was assessed using bioimpedance values measured with a body impedance analyzer (BIA 2000-S; Data Input GmbH, Frankfurt, Germany) [18]. Calibration of the BIA device occurred every day before and after the last use of the device with the test resistor. Results of the calibration were systematically documented and compared to target values of resistance: $\mathrm{R}=500 \Omega( \pm 4)$ and reactance $\mathrm{Xc}=144 \Omega( \pm 4)$. The multiple regression prediction equation developed by Janssen et al. was applied to estimate skeletal muscle mass [19]. Muscle mass index $\left(\mathrm{kg} / \mathrm{m}^{2}\right)$ was then calculated as skeletal muscle mass divided by height squared. For low muscle mass index, we used cut-offs previously published in KORA-Age: $\leq 8.72 \mathrm{~kg} / \mathrm{m}^{2}$ in men and $\leq 6.33 \mathrm{~kg} / \mathrm{m}^{2}$ in women [20]. 
Handgrip strength (kg) was assessed using the JAMAR handheld dynamometer (Saehan Corp., Masan, Korea). The mean value of three measurements in the participant's dominant hand was taken for analyses. We applied the EWGSOP cut-offs for low grip strength: $<30 \mathrm{~kg}$ in men and $<20 \mathrm{~kg}$ in women [17].

Gait speed (m/sec), assessed using the GAITRite system (CIR Systems, Havertown, PA, USA) [21], was used as the primary measure of physical performance. The standard cut-off of $\leq 0.8 \mathrm{~m} / \mathrm{sec}$ of the normal walking speed was applied for defining slow gait speed [17]. We also repeated all analyses using the time to complete the Timed Up and Go (TUG) test as an alternative measure of physical performance. The TUG test (sec) measured the time taken to stand up from a standard chair, walk a distance of $3 \mathrm{~m}$, turn, walk back to the chair and sit down. More time needed to complete the TUG test was defined as completing the test in $\geq 13.74 \mathrm{sec}$. This cut-point corresponds to the same proportion of individuals from the total baseline sample below $\leq 0.8 \mathrm{~m} / \mathrm{sec}$ for the gait speed (14.3\%).

\section{Serum 25OHD and PTH levels}

Non-fasting blood samples were taken at baseline between February and November 2009 at the KORA study center, usually between 07:30 am and 11:00 am, and drawn into serum gel S-Monovette tubes (Sarstedt, Nümbrecht, Germany). Blood was gently inverted twice and rested for $30 \mathrm{~min}$ at room temperature until complete coagulation. After centrifugation at $15^{\circ} \mathrm{C}$ for $10 \mathrm{~min}$, the serum obtained was aliquoted into Nunc cryotubes (Thermo Fisher Scientific, Waltham, MA, USA). For the analysis of both vitamin D and PTH status, serum probes were frozen at $-80^{\circ} \mathrm{C}$ at the KORA study center, transported on ice and stored at a minimum of $-80^{\circ} \mathrm{C}$ until analysis in partner laboratories. Serum concentrations of $25 \mathrm{OHD}$ and PTH were measured by an electrochemiluminescence immunoassay (ECLIA) using the Vitamin D total test (Elecsys 2011, Roche, Germany) and the intact PTH (1-84) test (Elecsys 2011, Roche, Germany), respectively. Intra- and inter-assay coefficients of variations were $<5 \%$ and $<10 \%$ for $25 \mathrm{OHD}$, and $<5 \%$ and $<5 \%$ for PTH, respectively. Vitamin D status was categorized as deficiency $(<25$ $\mathrm{nmol} / \mathrm{L})$, insufficiency $(25-<50 \mathrm{nmol} / \mathrm{L})$ and sufficiency $(\geq 50 \mathrm{nmol} / \mathrm{L}$, reference) [22]. Due to the lack of consistent reference ranges [23], PTH levels were categorized as $1^{\text {st }}$ tertile $(<2.8 \mathrm{pmol} / \mathrm{L}$, reference $), 2^{\text {nd }}$ tertile $(2.8-<3.8 \mathrm{pmol} / \mathrm{L})$ and $3^{\text {rd }}$ tertile $(\geq 3.8 \mathrm{pmol} / \mathrm{L})$.

\section{Confounders}

To avoid bias by adding covariates to the model that are intermediate factors or common effects, we constructed a directed acyclic graph (DAG) to identify relevant confounders in the association of vitamin D status with sarcopenia [24]. Using the graphical tool DAGitty [25], we identified a minimal adjustment set of covariates needed to estimate the causal effect without confounding: sex, age, Nutrition Score (SCREEN II), physical activity, BMI and use of vitamin D supplements. The variables — sex and age - were collected using the short form of the Demographics Standards of the German Society of Epidemiology [26]. The Nutrition Score, indicating risk of general malnutrition, was calculated using the German short form of the SCREEN II (Seniors in the Community: Risk Evaluation for Eating and Nutrition, version II) questionnaire [27]. Participants were classified as "low risk" (score 41-48), "moderate risk" (score 36-<41) or "high risk of malnutrition" (score <36). Physical activity assessment included frequency and duration of activity in summer and winter [28]. Possible answers were (1) $>2$ hours/week, (2) 1-2 hours/week, (3) <1 hour/week and (4) none. Participants, who had a total score $<5$, obtained by summing the numbers (1)-(4) relating to winter and summer, were classified to be "physically active", all others were classified as "inactive". Body mass index (BMI) was defined as body weight (kg) divided by height-squared $\left(\mathrm{m}^{2}\right)$. Use of supplements ingested in the last seven days was collected through a database supported computer software (IDOM, Instrument for Databased Assessment Of Medication) [29], together with the mode, dosage and frequency of ingestion [30]. Vitamin D supplement use was classified as "regular intake" or "no/irregular intake". The DAG with references can be seen in the Online Resource Fig. 1 (available in Calcified Tissue International online). Methods used to collect and categorize other baseline - not DAG confounders - variables (e.g. alcohol intake, smoking status, polypharmacy) have been described elsewhere [31]. 


\section{Statistical analysis}

Baseline descriptive characteristics were expressed as mean \pm standard deviation (SD), median $\left(1^{\text {st }}\right.$ quartile$3^{\text {rd }}$ quartile) or proportion (\%) by sarcopenia status at baseline and were compared across categories using one-way ANOVA or Kruskal-Wallis tests for continuous variables and Pearson's chi-squared tests for categorical variables.

In cross-sectional analyses $(n=975)$, multiple logistic regression analyses tested the associations of categorized baseline 25OHD levels with the prevalence of sarcopenia and related muscle parameters, and in prospective analyses $(n=702)$ with the incidence of sarcopenia and related muscle parameters in individuals without the respective condition at baseline. We repeated the prospective analyses using a combined endpoint of incident sarcopenia or death, assuming that many of those who died before the follow-up examination developed sarcopenia prior to death. Results were expressed as odds ratios (OR) with $95 \%$ confidence interval (CI). Annual change (\%) in each muscle parameter was calculated as: [(follow-upbaseline)/baseline $)^{*}(100 /$ time $\left.)\right]$. Multiple linear regression analyses tested the associations of baseline 25 OHD with annual changes in muscle parameters. Results were expressed as $\beta$-coefficients with $95 \%$ CI. In all analyses, three adjusted models were fitted: Model 1 was adjusted for age and sex, Model 2 was fullyadjusted for DAG confounders: age, sex, Nutrition Score (SCREEN II), physical activity, BMI and use of vitamin D supplements, and Model 3 was fully-adjusted plus additional inclusion of PTH tertiles to test the potential mediating effect of PTH. A $p$-value $<0.05$ was considered statistically significant. All statistical analyses were performed using the statistical software package, SAS version 9.4 (SAS Institute Inc., Cary, NC, USA).

\section{Results}

\section{Participant characteristics}

Baseline characteristics of the 975 individuals ( $49.2 \%$ female, mean age $75.7 \pm 6.5$ years) included in crosssectional analyses are shown in Table 1 stratified by sarcopenia status.

Overall, $6.7 \%$ of individuals had prevalent sarcopenia (2.3\% in men, $4.4 \%$ in women). When using the TUG test as an alternative to gait speed, the prevalence of sarcopenia minimally decreased to $6.6 \%$ (2.3\% in men, $4.3 \%$ in women). Sarcopenic individuals were more likely to be female, older, to live alone, be divorced or widowed, to be physically inactive, to have a lower BMI and lower 25OHD levels. 
Table 1 Participant characteristics by sarcopenia status at baseline, $n=975$

\begin{tabular}{|c|c|c|c|c|c|c|c|}
\hline Socio-demographic factors & \multicolumn{2}{|c|}{$\begin{array}{c}\text { All } \\
n=975\end{array}$} & \multicolumn{2}{|c|}{$\begin{array}{c}\text { No sarcopenia } \\
n=910\end{array}$} & \multicolumn{2}{|c|}{$\begin{array}{c}\text { Sarcopenia } \\
n=65\end{array}$} & $\begin{array}{c}p- \\
\text { value }\end{array}$ \\
\hline Women, \% & 480 & $(49.2)$ & 437 & $(48.0)$ & 43 & $(66.2)$ & 0.005 \\
\hline Age, years & 75.7 & \pm 6.5 & 75.3 & \pm 6.4 & 81.0 & \pm 6.4 & $<0.001$ \\
\hline Categorized, \% & & & & & & & $<0.001$ \\
\hline $65-74$ & 434 & $(44.5)$ & 422 & $(46.4$ & 12 & $(18.5)$ & \\
\hline $75-84$ & 435 & $(44.6)$ & 403 & $(44.3$ & 32 & $(49.2)$ & \\
\hline $85+$ & 106 & $(10.9)$ & 85 & $(9.3$ & 21 & $(32.3)$ & \\
\hline Family status, \% & & & & & & & $<0.001$ \\
\hline Living with a partner & 617 & $(63.8)$ & 590 & $(65.2)$ & 27 & $(43.6)$ & \\
\hline Living alone, divorced or widowed & 350 & $(36.2)$ & 315 & $(34.8)$ & 35 & $(56.5)$ & \\
\hline Low educational level, \% & 195 & $(20.0)$ & 183 & $(20.1)$ & 12 & $(18.5)$ & 0.748 \\
\hline \multicolumn{8}{|l|}{ Lifestyle factors } \\
\hline Nutrition Score (SCREEN II), \% & & & & & & & 0.317 \\
\hline Low risk (41 to 48 ) & 381 & $(39.1)$ & 360 & $(39.6)$ & 21 & $(32.3)$ & \\
\hline Medium risk (36 to $<41)$ & 350 & $(35.9)$ & 327 & $(35.9)$ & 23 & $(35.4)$ & \\
\hline High risk $(<36)$ & 244 & $(25.0)$ & 223 & $(24.5)$ & 21 & $(32.3)$ & \\
\hline Physically inactive, \% & 442 & $(45.3)$ & 404 & $(44.4)$ & 38 & $(58.5)$ & 0.028 \\
\hline Alcohol consumption ${ }^{\mathrm{a}}, \%$ & & & & & & & 0.008 \\
\hline Abstainer & 340 & $(34.9)$ & 306 & $(33.7)$ & 34 & $(52.3)$ & \\
\hline Light-moderate drinker & 500 & $(51.3)$ & 477 & $(52.5)$ & 23 & $(35.4)$ & \\
\hline High drinker & 134 & $(13.8)$ & 126 & $(13.9)$ & 8 & $(12.3)$ & \\
\hline Smoking, \% & & & & & & & 0.286 \\
\hline Never smoker & 555 & $(56.9)$ & 512 & $(56.3)$ & 43 & $(66.2)$ & \\
\hline Ex-smoker & 373 & $(38.3)$ & 353 & $(38.8)$ & 20 & $(30.7)$ & \\
\hline Current smoker & 47 & $(4.8)$ & 45 & $(5.0)$ & 2 & $(3.1)$ & \\
\hline \multicolumn{8}{|l|}{ Health factors } \\
\hline BMI, $\mathrm{kg} / \mathrm{m}^{2}$ & 28.5 & \pm 4.3 & 28.7 & \pm 4.2 & 24.9 & \pm 3.1 & $<0.001$ \\
\hline Polypharmacy ( $\geq 5$ medications), $\%$ & 304 & $(31.2)$ & 284 & $(31.2)$ & 20 & $(30.8)$ & 0.941 \\
\hline $\mathrm{eGFR}<60 \mathrm{~mL} / \mathrm{min} / 1.73 \mathrm{~m}^{2 \mathrm{~b}}, \%$ & 381 & $(39.1)$ & 351 & $(38.6)$ & 30 & $(46.2)$ & 0.229 \\
\hline Multimorbidity ${ }^{\mathrm{c}}, \%$ & & & & & & & 0.259 \\
\hline No disease & 89 & $(9.2)$ & 86 & $(9.5)$ & 3 & $(4.7)$ & \\
\hline One disease & 251 & $(25.9)$ & 237 & $(26.2)$ & 14 & $(21.9)$ & \\
\hline Two or more diseases & 629 & $(64.9)$ & 582 & $(64.3)$ & 47 & $(73.4)$ & \\
\hline Vitamin D supplements, \% & & & & & & & 0.500 \\
\hline Regular use & 132 & $(13.5)$ & 125 & $(13.7)$ & 7 & $(10.8)$ & \\
\hline No/irregular use & 843 & $(86.5)$ & 785 & $(86.3)$ & 58 & $(89.2)$ & \\
\hline Calcium supplements, \% & & & & & & & 0.642 \\
\hline Regular use & 139 & $(14.3)$ & 131 & $(14.4)$ & 8 & $(12.3)$ & \\
\hline No/irregular use & 836 & $(85.7)$ & 779 & $(85.6)$ & 57 & $(87.7)$ & \\
\hline \multicolumn{8}{|l|}{ Biological measures } \\
\hline Season of blood collection, \% & & & & & & & 0.278 \\
\hline Jun-Aug & 341 & $(35.0)$ & 324 & $(35.6)$ & 17 & $(26.2)$ & \\
\hline Sep-Nov & 215 & $(22.1)$ & 200 & $(22.0)$ & 15 & $(23.1)$ & \\
\hline
\end{tabular}




\begin{tabular}{|c|c|c|c|c|c|c|c|}
\hline Feb-May & 419 & $(43.0)$ & 386 & $(42.4)$ & 33 & $(50.8)$ & \\
\hline 25OHD, nmol/L & 48.9 & $31.9-69.9$ & 49.0 & $32.7-70.6$ & 41.4 & $24.4-67.1$ & 0.025 \\
\hline Categorized, \% & & & & & & & 0.002 \\
\hline Sufficiency $(\geq 50)$ & 468 & $(48.0)$ & 443 & (48.7) & 25 & $(38.5)$ & \\
\hline Insufficiency $(25-<50)$ & 369 & $(37.9)$ & 348 & $(38.2)$ & 21 & $(32.3)$ & \\
\hline Deficiency $(<25)$ & 138 & $(14.2)$ & 119 & $(13.1)$ & 19 & $(29.2)$ & \\
\hline PTH, pmol/L & 3.3 & $2.5-4.1$ & 3.3 & $2.5-4.1$ & 3.1 & $2.5-4.3$ & 0.923 \\
\hline Categorized, \% & & & & & & & 0.466 \\
\hline $1^{\text {st }}$ tertile $(<2.8)$ & 326 & $(33.4)$ & 302 & $(33.2)$ & 24 & $(36.9)$ & \\
\hline $2^{\text {nd }}$ tertile $(2.8-<3.8)$ & 323 & $(33.1)$ & 306 & (33.6) & 17 & $(26.2)$ & \\
\hline $3^{\text {rd }}$ tertile $(\geq 3.8)$ & 326 & $(33.4)$ & 302 & $(33.2)$ & 24 & $(36.9)$ & \\
\hline Hyperparathyroidism ( $\geq 6.8)$, \% & 55 & $(5.6)$ & 50 & $(5.5)$ & 5 & (7.7) & 0.458 \\
\hline \multicolumn{8}{|l|}{ Sarcopenia-related muscle parameters } \\
\hline Muscle mass index ${ }^{\mathrm{d}}, \mathrm{kg} / \mathrm{m}^{2}$ & 8.8 & \pm 1.7 & 8.9 & \pm 1.6 & 6.6 & \pm 1.3 & $<0.001$ \\
\hline Grip strength, kg & 26.8 & \pm 9.7 & 27.5 & \pm 9.6 & 17.9 & \pm 6.6 & $<0.001$ \\
\hline Gait speed $^{\mathrm{e}}, \mathrm{m} / \mathrm{sec}$ & 1.1 & \pm 0.2 & 1.1 & \pm 0.2 & 1.0 & \pm 0.2 & 0.0311 \\
\hline Walking aid $^{\mathrm{f}} \%$ & 45 & $(5.0)$ & 43 & $(5.1)$ & 2 & (3.9) & 0.695 \\
\hline Time to complete the TUG test ${ }^{\mathrm{g}}$, sec & 9.8 & $8.5-11.9$ & 9.7 & $8.5-11.9$ & 10.6 & $9.2-12.5$ & 0.060 \\
\hline
\end{tabular}

Results expressed as mean $\pm \mathrm{SD}$, median ( $1^{\text {st }}$ quartile- $3^{\text {rd }}$ quartile) or proportion (\%)

Number of missing values: ${ }^{\mathrm{a}} 1 ;{ }^{\mathrm{b}} 1 ;{ }^{\mathrm{c}} 6 ;{ }^{\mathrm{d}} 18 ;{ }^{\mathrm{e}} 74 ;{ }^{\mathrm{f}} 74 ;{ }^{\mathrm{g}} 62$

25OHD, 25-hydroxyvitamin D; PTH, parathyroid hormone; TUG test, Timed Up and Go test; standard deviation (SD)

\section{Cross-sectional results}

At baseline, individuals with low baseline $25 \mathrm{OHD}<25 \mathrm{vs} . \geq 50 \mathrm{nmol} / \mathrm{L}$ tended to have higher odds of prevalent sarcopenia in fully-adjusted analysis (OR (95\%CI): 2.14 (0.98-4.63)). Additional inclusion of PTH tertiles strengthened the association, which became significant (OR (95\%CI): 2.64 (1.16-5.98)). Vitamin D deficiency was associated with higher odds of low grip strength, slow gait speed and more time needed for the TUG test after multivariable adjustment (see Table 1 in the Online Resource, available in Calcified Tissue International online).

\section{Prospective results}

Annual changes in muscle parameters are shown in Table 2 stratified by baseline 25OHD levels. In fully-adjusted analysis, individuals with low baseline $25 \mathrm{OHD}<25 \mathrm{vs} . \geq 50 \mathrm{nmol} / \mathrm{L}$ had a $0.94 \%$ greater annual decrease in muscle mass index and a $3.06 \%$ greater annual increase in time to complete the TUG test. Vitamin D status was not significantly associated with change in grip strength or gait speed.

After $2.9 \pm 0.1$ years of follow-up, $4.3 \%$ of individuals without sarcopenia at baseline developed incident sarcopenia ( $2.8 \%$ in men; $5.8 \%$ in women). The incidence of sarcopenia remained unchanged when using the TUG test as an alternative to gait speed. Lower baseline 25OHD levels were not significantly associated with the risk for developing incident sarcopenia. Adjustment for PTH tertiles slightly attenuated the observed trends (Table 3). When repeating analyses using a combined endpoint of incident sarcopenia or death, $11.5 \%$ of individuals developed the outcome. The presence of both vitamin D deficiency and insufficiency were strongly associated with a higher risk for incident sarcopenia or death (deficiency: OR (95\% CI): 3.19 (1.546.57); insufficiency: OR (95\% CI): 1.84 (1.06-3.22)). Vitamin D insufficiency was associated with a higher risk for developing low muscle mass index after multivariable adjustment (Table 3 ). 
Table 2 Prospective associations of baseline 25OHD levels with annual changes in related muscle parameters, $n=702$

\begin{tabular}{|c|c|c|c|c|c|c|}
\hline & \multirow{2}{*}{\multicolumn{3}{|c|}{$\beta(95 \% \mathrm{CI})$}} \\
\hline & & & & & & \\
\hline $\begin{array}{l}\text { Outcome at follow-up } \\
\text { (continuous) }\end{array}$ & 25OHD & $\begin{array}{c}\% \text { change per year } \\
\text { of outcome, mean }( \pm \mathrm{SD})\end{array}$ & $n$ & $\begin{array}{c}\text { Model 1: } \\
\text { Age and sex }\end{array}$ & $\begin{array}{c}\text { Model 2: } \\
\text { Fully-adjusted }\end{array}$ & $\begin{array}{c}\text { Model 3: } \\
\text { Model 2 + PTH }\end{array}$ \\
\hline \multirow{4}{*}{ Muscle mass index } & All & $-0.17 \%( \pm 2.28)$ & 681 & & & \\
\hline & Deficiency & $-0.89 \%( \pm 2.98)$ & 75 & $-1.03(-1.61,-0.45) * *$ & $-0.94(-1.55,-0.34) * *$ & $-0.92(-1.54,-0.30)^{*}$ \\
\hline & Insufficiency & $-0.40 \%( \pm 2.29)$ & 262 & $-0.52(-0.89,-0.15) * *$ & $-0.47(-0.86,-0.09) *$ & $-0.46(-0.84,-0.07)^{*}$ \\
\hline & Sufficiency & $0.15 \%( \pm 2.04)$ & 344 & $0.00(r e f)$ & $0.00($ ref $)$ & $0.00(r e f)$ \\
\hline \multirow{4}{*}{ Grip strength } & All & $-0.42 \%( \pm 7.16)$ & 702 & & & \\
\hline & Deficiency & $-1.10 \%( \pm 7.38)$ & 76 & $0.03(-1.79,1.85)$ & $0.34(-1.58,2.25)$ & $0.46(-1.48,2.41)$ \\
\hline & Insufficiency & $-0.12 \%( \pm 7.76)$ & 267 & $0.57(-0.57,1.72)$ & $0.71(-0.48,1.91)$ & $0.72(-0.48,1.93)$ \\
\hline & Sufficiency & $-0.50 \%( \pm 6.64)$ & 359 & $0.00(r e f)$ & $0.00($ ref $)$ & $0.00($ ref $)$ \\
\hline \multirow{4}{*}{ Gait speed } & All & $2.95 \%( \pm 8.12)$ & 590 & & & \\
\hline & Deficiency & $2.20 \%( \pm 6.75)$ & 54 & $-0.65(-3.07,1.76)$ & $-0.71(-3.23,1.80)$ & $-0.54(-3.08,2.00)$ \\
\hline & Insufficiency & $3.27 \%( \pm 8.77)$ & 218 & $0.58(-0.84,2.00)$ & $0.64(-0.83,2.11)$ & $0.75(-0.73,2.23)$ \\
\hline & Sufficiency & $2.86 \%( \pm 7.86)$ & 318 & $0.00(r e f)$ & $0.00(r e f)$ & $0.00(r e f)$ \\
\hline \multirow{4}{*}{$\begin{array}{c}\text { Time to complete the TUG } \\
\text { test }\end{array}$} & All & $-0.19 \%( \pm 8.36)$ & 612 & & & \\
\hline & Deficiency & $3.29 \%( \pm 13.28)$ & 58 & $3.47(1.14-5.81)^{* *}$ & $3.06(0.63-5.49) *$ & $3.06(0.61-5.51)^{* *}$ \\
\hline & Insufficiency & $0.68 \%( \pm 8.05)$ & 229 & $1.49(0.11-2.88)^{*}$ & $1.42(-0.02-2.85)$ & $1.53(0.09-2.98) *$ \\
\hline & Sufficiency & $-1.42 \%( \pm 7.14)$ & 325 & $0.00(r e f)$ & $0.00(r e f)$ & $0.00(r e f)$ \\
\hline
\end{tabular}

Results of multiple linear regression analyses expressed as $\beta$ ( $95 \% \mathrm{CI})$. $\beta$ coefficient is the mean difference in percentage change per year. Model $\mathbf{1}$, adjusted for sex and age; Model $\mathbf{2}$, fullyadjusted for DAG confounders: sex, age, Nutrition Score (SCREEN II), physical activity, BMI and use of vitamin D supplements; Model 3, fully-adjusted plus PTH tertiles; * $\boldsymbol{p}<\mathbf{0 . 0 5}$ vs. ref, $* * p<0.01$ vs. $r e f$

25OHD, 25-hydroxyvitamin D categorized as deficiency ( $<25 \mathrm{nmol} / \mathrm{L})$, insufficiency $(25-<50 \mathrm{nmol} / \mathrm{L})$ and sufficiency ( $\geq 50 \mathrm{nmol} / \mathrm{L}$, ref); PTH, parathyroid hormone; TUG test, Timed Up and Go test; DAG, directed acyclic graph; OR, odds ratio; CI, confidence interval; SD, standard deviation 
Table 3 Prospective associations of baseline 25OHD levels with the incidence of sarcopenia (or death) and related muscle parameters, $n=702$

\begin{tabular}{|c|c|c|c|c|c|c|}
\hline & \multirow{2}{*}{\multicolumn{3}{|c|}{ OR (95 \% CI) }} \\
\hline & & & & & & \\
\hline $\begin{array}{l}\text { Outcome at follow-up } \\
\text { (dichotomized) }\end{array}$ & 25OHD & $\begin{array}{c}\text { Incidence } \\
\text { of outcome }\end{array}$ & $n$ & $\begin{array}{c}\text { Model 1: } \\
\text { Age and sex }\end{array}$ & $\begin{array}{c}\text { Model 2: } \\
\text { Fully-adjusted }\end{array}$ & $\begin{array}{c}\text { Model 3: } \\
\text { Model 2 + PTH }\end{array}$ \\
\hline \multirow{4}{*}{ Sarcopenia } & All & $4.3 \%$ & 702 & & & \\
\hline & Deficient & $5.3 \%$ & 76 & $1.10(0.29-3.44)$ & $2.38(0.54-8.99)$ & $1.91(0.43-7.24)$ \\
\hline & Insufficiency & $5.2 \%$ & 267 & $1.35(0.61-3.05)$ & $2.10(0.89-5.04)$ & $1.98(0.83-4.83)$ \\
\hline & Sufficiency & $3.3 \%$ & 359 & $1.00($ ref $)$ & $1.00(r e f)$ & $1.00($ ref $)$ \\
\hline \multirow{4}{*}{ Sarcopenia or death } & All & $11.5 \%$ & 759 & & & \\
\hline & Deficiency & $23.4 \%$ & 94 & $2.44(1.25-4.69)^{* *}$ & $3.19(1.54-6.57)^{* * *}$ & $2.95(1.40-6.18)^{* *}$ \\
\hline & Insufficiency & $12.5 \%$ & 289 & $1.51(0.89-2.57)$ & $1.84(1.06-3.22) *$ & $1.77(1.01-3.12) *$ \\
\hline & Sufficiency & $7.7 \%$ & 376 & $1.00($ ref $)$ & $1.00(\mathrm{ref})$ & $1.00($ ref $)$ \\
\hline \multirow{4}{*}{ Low muscle mass index } & All & $7.8 \%$ & 681 & & & \\
\hline & Deficiency & $8.0 \%$ & 75 & $0.97(0.34-2.42)$ & $2.52(0.76-7.59)$ & $2.39(0.72-7.30)$ \\
\hline & Insufficiency & $9.2 \%$ & 262 & $1.24(0.67-2.27)$ & $2.01(1.02-4.01)^{*}$ & $1.94(0.98-3.90)$ \\
\hline & Sufficiency & $6.7 \%$ & 344 & $1.00(\mathrm{ref})$ & $1.00(r e f)$ & $1.00(r e f)$ \\
\hline \multirow{4}{*}{ Low grip strength } & All & $34.2 \%$ & 702 & & & \\
\hline & Deficiency & $44.7 \%$ & 76 & $1.14(0.63-2.01)$ & $1.07(0.58-1.95)$ & $0.99(0.53-1.82)$ \\
\hline & Insufficiency & $38.2 \%$ & 267 & $1.21(0.84-1.74)$ & $1.17(0.80-1.71)$ & $1.13(0.77-1.66)$ \\
\hline & Sufficiency & $29.0 \%$ & 359 & $1.00(\mathrm{ref})$ & $1.00(\mathrm{ref})$ & $1.00(r e f)$ \\
\hline \multirow{4}{*}{ Slow gait speed } & All & $6.8 \%$ & 590 & & & \\
\hline & Deficiency & $13.0 \%$ & 54 & $2.05(0.68-5.64)$ & $1.67(0.53-4.85)$ & $1.54(0.51-4.91)$ \\
\hline & Insufficiency & $8.7 \%$ & 218 & $1.66(0.80-3.52)$ & $1.53(0.71-3.40)$ & $1.50(0.68-3.38)$ \\
\hline & Sufficiency & $4.4 \%$ & 318 & $1.00(r e f)$ & $1.00(r e f)$ & $1.00($ ref $)$ \\
\hline \multirow{4}{*}{$\begin{array}{c}\text { More time needed to complete } \\
\text { the TUG test }\end{array}$} & All & $10.3 \%$ & 612 & & & \\
\hline & Deficiency & $20.7 \%$ & 58 & $2.71(1.15-6.16)$ & $2.02(0.82-4.83)$ & $1.94(0.77-4.70)$ \\
\hline & Insufficiency & $13.1 \%$ & 229 & $1.83(1.01-3.39)$ & $1.57(0.82-3.02)$ & $1.53(0.79-2.97)$ \\
\hline & Sufficiency & $6.5 \%$ & 325 & $1.00($ ref $)$ & $1.00($ ref $)$ & $1.00(\mathrm{ref})$ \\
\hline
\end{tabular}

Results of multiple logistic regression analyses expressed as OR (95\% CI): Model 1, adjusted for sex and age; Model 2, fully-adjusted for DAG confounders: sex, age, Nutrition Score (SCREEN II), physical activity, BMI and use of vitamin D supplements; Model 3, fully-adjusted plus PTH tertiles; *p $<<\mathbf{0 . 0 5}$ vs. ref, **p $<\mathbf{0 . 0 1}$ vs. $\boldsymbol{r e f}$

25OHD, 25-hydroxyvitamin D categorized as deficiency ( $<25 \mathrm{nmol} / \mathrm{L})$, insufficiency $(25-<50 \mathrm{nmol} / \mathrm{L})$ and sufficiency $(\geq 50 \mathrm{nmol} / \mathrm{L}$, ref); PTH, parathyroid hormone; TUG test, Timed Up and Go test; DAG, directed acyclic graph; OR, odds ratio; CI, confidence interva 


\section{Discussion}

In this three-year prospective study among German older adults, low baseline $25 \mathrm{OHD}<25 v s$. $\geq 50 \mathrm{nmol} / \mathrm{L}$ were significantly associated with changes in muscle mass and physical performance as assessed by the time to complete the TUG test. Vitamin D status was not associated with the risk for developing incident sarcopenia. However, when including death as a combined outcome alongside incident sarcopenia, there was a strong positive association with low baseline $25 \mathrm{OHD}<25 v s$. $\geq 50 \mathrm{nmol} / \mathrm{L}$. There was no evidence for a mediating effect of PTH.

\section{OHD and changes in muscle mass and physical performance}

In previous prospective studies, low $25 \mathrm{OHD}$ levels were not linked to change in muscle mass [3,5,6]. Some studies have shown, however, an association with loss of muscle strength $[5,6,8]$. Findings on the association between $25 \mathrm{OHD}$ and decline in gait speed have relatively consistently shown no significant association $[3,10,11]$. In our study, gait speed increased with a mean annual gain of $2.95 \pm 8.12 \%$, possibly due to the selection bias in KORAAge, whereby more healthy individuals participated at follow-up [32]. However, caution is warranted when interpreting our gait speed variable because a comparable effect was not seen for the TUG test. Studies investigating vitamin D in relation to change in other performance tests, including the TUG test, have shown either no association [9-11] or a greater decline in physical performance among those with low baseline 25OHD $[7,33]$.

Meta-analyses of randomized controlled trials (RCTs) have shown heterogeneous effects of vitamin D supplementation on muscle parameters. One meta-analysis of 13 trials in older adults aged $\geq 60$ years has shown evidence of decreased time to complete the TUG test and gains in lower extremity strength, but no beneficial effect on gait speed [34]. Another larger meta-analysis of 30 trials in individuals with mean age 61.1 years found a small positive effect on muscle strength but not on muscle mass [35]. More recently, a meta-analysis of 15 studies in individuals aged $\geq 65$ years demonstrated no improvement in muscle strength after vitamin $\mathrm{D}$ administration [36]. Further RCTs that compare effects of vitamin D supplementation in sarcopenic vs. nonsarcopenic participants are needed to confirm the place of vitamin D supplementation in the management of sarcopenia, and to clarify optimal $25 \mathrm{OHD}$ levels for muscle health.

\section{OHD and incident sarcopenia or death}

Very few prospective studies have examined vitamin D status in relation to incident sarcopenia. In one study conducted among 433 men aged $\geq 60$ years, the risk for developing incident sarcopenia over 4.3 years was not significantly related to baseline 25OHD in multivariable analysis [3]. Another study following a cohort of 709 men aged $\geq 70$ years over 5 years showed that baseline $25 \mathrm{OHD}<40 v s . \geq 68.9 \mathrm{nmol} / \mathrm{L}$ were significantly associated with increased odds of incident sarcopenia with an OR (95\% CI) of 2.53 (1.14-5.64) [4]. Comparison of our results with the findings of these prospective studies are limited due to differences in study populations (only men), sarcopenia definitions, length of follow-up, cut-off values to define low baseline 25OHD levels, and due to diverging confounding adjustment.

A large problem of prospective studies among older cohorts are competing risks, with numerous losses to followup due to death. In a previous KORA-Age analysis, low vitamin D status was associated with higher mortality [37]. Assuming that older individuals may often develop sarcopenia before death [38,39], we tried to address the problem of competing risks by means of a combined endpoint, which included incident cases of sarcopenia and all losses to follow-up due to death. We found a strong positive association of low baseline $25 \mathrm{OHD}<25 v s$. $\geq 50$ $\mathrm{nmol} / \mathrm{L}$ with this combined outcome, highlighting the importance of considering competing risks such as mortality in older cohorts. Future longitudinal studies should include repeated follow-up examinations after short time periods to identify individuals who develop incident sarcopenia before death. 


\section{Possible mechanisms, including PTH}

Mechanistically, a direct effect of the biologically active form of vitamin D on muscle has been suggested following the localization of a vitamin D receptor expressed on human muscle tissue [13]. Other studies have explored the well-known inverse relationship between serum 25OHD and PTH, showing effects of increased PTH levels on skeletal muscle mass and function [6], and suggesting that hyperparathyroidism secondary to vitamin D deficiency may mediate the effect of vitamin D on muscle [40].

In the present study, adjustment for PTH tertiles strengthened the association of 25OHD with sarcopenia in crosssectional analyses, but only slightly attenuated it in prospective analyses. This suggests that a meaningful mediating effect of PTH was unlikely, though the number of participants with hyperparathyroidism was very small. Of note, there may be other indirect biological pathways mediating the effect of vitamin $\mathrm{D}$ on muscle, including hypocalcemia [14] or hypophosphatemia [15]. Further mechanistic studies are required to better understand the mechanisms by which 25OHD levels may influence sarcopenia and its onset.

\section{Strengths and limitations}

Strengths include the use of a large, broadly representative, sample of community-dwelling German older adults with prospective data. Because reasons for non-participation were systematically recorded, we were able to incorporate withdrawal due to death as a combined outcome with incident sarcopenia. We have included 25OHD levels in addition to a wide range of covariates trough DAG modeling, thereby minimizing confounding in examining the causal association between vitamin D status and sarcopenia. Nonetheless residual confounding cannot be entirely excluded. Furthermore, we could not account for the effects of change in 25OHD levels on change in muscle parameters, because 25OHD levels were only measured at baseline. The power of the present study was limited especially regarding analyses on incident sarcopenia due to the low incidence in our rather healthy study population. While the EWGSOP allows use of BIA in conjunction with the Janssen multiple regression prediction equation for estimation of the skeletal muscle mass [17], we recognize that this approach should be used with caution because estimates of skeletal muscle mass can be artificially elevated due to altered hydration, including fluid accumulation and hypohydration [41]. . Furthermore, PTH levels are subject to diurnal rhythms and influenced by the ingestion of calcium-containing foods, hence PTH results may have been affected by the non-fasting status of KORA-Age participants and possible batch effects associated with sampling across extended time periods. Accurate interpretation and comparison of PTH results across studies may also be hampered by the lack of standardization and robust reference ranges due to variable analytical methods used for its determination [23].

\section{Conclusion}

In conclusion, our finding suggests that a low vitamin D status may be an early risk factor for changes in muscle mass and physical performance in older adults. Replenishing 25OHD levels may be important for the preservation of specific sarcopenia-related muscle parameters but further randomized trials are needed to assess whether the observed associations are causal and to determine optimal 25OHD levels for muscle health. We could not demonstrate a statistically significant association of low vitamin D status with incident sarcopenia, but a significant association was found with the combined endpoint of sarcopenia and death. This highlights the need for future well-designed prospective studies that address the issue of competing risks such as mortality in older cohorts.

Acknowledgments We thank all participants of the KORA-Age study for their extraordinary commitment and goodwill. The KORA study was initiated and financed by the Helmholtz Zentrum München - German Research Center for Environmental Health, which is funded by the German Federal Ministry of Education and Research (BMBF) and by the State of Bavaria. The KORA-Age project was financed by the German Federal Ministry of Education and Research (BMBF FKZ 01ET0713 and 01ET1003A) as part of the 'Health in old age' program. RC is supported by a $\mathrm{PhD}$ scholarship from the 'Studienstiftung des deutschen Volkes'. The funders had no role in study design, data collection and analysis, decision to publish, or preparation of the manuscript. 
Authors' contribution RC and BT conceived and designed the study. RC analyzed the data and wrote the first draft of the paper. EV, HBF and BT, as members of the PhD Thesis Committee of RC, helped in interpreting the data. All authors revised the paper critically for intellectual content and approved the final version. All authors agree to be accountable for the work and to ensure that any questions relating to the accuracy and integrity of the paper are investigated and properly resolved.

\section{Compliance with Ethical Standards}

Conflicts of interest Romy Conzade, Eva Grill, Heike A. Bischoff-Ferrari, Uta Ferrari, Alexander Horsch, Wolfgang Koenig, Annette Peters and Barbara Thorand declare that they have no conflict of interest.

Ethical Approval, Human Rights and Informed Consent The KORA-Age was approved by the Ethics Committee of the Bavarian Medical Association (reference number 08064). Written informed consent has been obtained from the participants and all investigations have been conducted according to the principles expressed in the Helsinki declaration.

\section{References}

1. Sayer, A.A.; Robinson, S.M.; Patel, H.P.; Shavlakadze, T.; Cooper, C.; Grounds, M.D. New horizons in the pathogenesis, diagnosis and management of sarcopenia. Age Ageing 2013, 42, 145-150.

2. Bischoff-Ferrari, H.A. Relevance of vitamin D in muscle health. Rev Endocr Metab Disord 2012, 13, 71-77.

3. Gielen, E.; O'Neill, T.W.; Pye, S.R.; Adams, J.E.; Wu, F.C.; Laurent, M.R.; Claessens, F.; Ward, K.A.; Boonen, S.; Bouillon, R., et al. Endocrine determinants of incident sarcopenia in middle-aged and elderly European men. J Cachexia Sarcopenia Muscle 2015, 6, 242-252.

4. Hirani, V.; Cumming, R.G.; Naganathan, V.; Blyth, F.; Le Couteur, D.G.; Hsu, B.; Handelsman, D.J.; Waite, L.M.; Seibel, M.J. Longitudinal Associations Between Vitamin D Metabolites and Sarcopenia in Older Australian men: The Concord Health and Aging in Men Project. J Gerontol A Biol Sci Med Sci 2017, 73, 131138.

5. Scott, D.; Blizzard, L.; Fell, J.; Ding, C.; Winzenberg, T.; Jones, G. A prospective study of the associations between 25 -hydroxy-vitamin D, sarcopenia progression and physical activity in older adults. Clin Endocrinol (Oxf) 2010, 73, 581-587.

6. Visser, M.; Deeg, D.J.; Lips, P. Low vitamin D and high parathyroid hormone levels as determinants of loss of muscle strength and muscle mass (sarcopenia): the Longitudinal Aging Study Amsterdam. J Clin Endocrinol Metab 2003, 88, 5766-5772.

7. $\quad$ Wicherts, I.S.; van Schoor, N.M.; Boeke, A.J.; Visser, M.; Deeg, D.J.; Smit, J.; Knol, D.L.; Lips, P. Vitamin D status predicts physical performance and its decline in older persons. J Clin Endocrinol Metab 2007, 92, 2058-2065.

8. $\quad$ Granic, A.; Hill, T.R.; Davies, K.; Jagger, C.; Adamson, A.; Siervo, M.; Kirkwood, T.B.; Mathers, J.C.; Sayer, A.A. Vitamin D Status, Muscle Strength and Physical Performance Decline in Very Old Adults: A Prospective Study. Nutrients 2017, 9.

9. Bartali, B.; Frongillo, E.A.; Guralnik, J.M.; Stipanuk, M.H.; Allore, H.G.; Cherubini, A.; Bandinelli, S.; Ferrucci, L.; Gill, T.M. Serum micronutrient concentrations and decline in physical function among older persons. Jama 2008, 299, 308-315.

10. Houston, D.K.; Tooze, J.A.; Neiberg, R.H.; Hausman, D.B.; Johnson, M.A.; Cauley, J.A.; Bauer, D.C.; Cawthon, P.M.; Shea, M.K.; Schwartz, G.G., et al. 25-hydroxyvitamin D status and change in physical performance and strength in older adults: the Health, Aging, and Body Composition Study. Am J Epidemiol 2012, 176, 1025-1034.

11. Verreault, R.; Semba, R.D.; Volpato, S.; Ferrucci, L.; Fried, L.P.; Guralnik, J.M. Low serum vitamin d does not predict new disability or loss of muscle strength in older women. J Am Geriatr Soc 2002, 50, 912-917. 12. Ceglia, L.; Harris, S.S. Vitamin D and its role in skeletal muscle. Calcif Tissue Int 2013, 92, $151-162$. 13. Ceglia, L.; da Silva Morais, M.; Park, L.K.; Morris, E.; Harris, S.S.; Bischoff-Ferrari, H.A.; Fielding, R.A.; Dawson-Hughes, B. Multi-step immunofluorescent analysis of vitamin D receptor loci and myosin heavy chain isoforms in human skeletal muscle. J Mol Histol 2010, 41, 137-142. 
14. Wassner, S.J.; Li, J.B.; Sperduto, A.; Norman, M.E. Vitamin D Deficiency, hypocalcemia, and increased skeletal muscle degradation in rats. J Clin Invest 1983, 72, 102-112.

15. Schubert, L.; DeLuca, H.F. Hypophosphatemia is responsible for skeletal muscle weakness of vitamin D deficiency. Arch Biochem Biophys 2010, 500, 157-161.

16. Peters, A.; Doring, A.; Ladwig, K.H.; Meisinger, C.; Linkohr, B.; Autenrieth, C.; Baumeister, S.E.; Behr, J.; Bergner, A.; Bickel, H., et al. [Multimorbidity and successful aging: the population-based KORA-Age study]. Z Gerontol Geriatr 2011, 44 Suppl 2, 41-54.

17. Cruz-Jentoft, A.J.; Baeyens, J.P.; Bauer, J.M.; Boirie, Y.; Cederholm, T.; Landi, F.; Martin, F.C.; Michel, J.P.; Rolland, Y.; Schneider, S.M., et al. Sarcopenia: European consensus on definition and diagnosis: Report of the European Working Group on Sarcopenia in Older People. Age Ageing 2010, 39, 412-423.

18. Kyle, U.G.; Genton, L.; Karsegard, L.; Slosman, D.O.; Pichard, C. Single prediction equation for bioelectrical impedance analysis in adults aged 20--94 years. Nutrition 2001, 17, 248-253.

19. Janssen, I.; Heymsfield, S.B.; Baumgartner, R.N.; Ross, R. Estimation of skeletal muscle mass by bioelectrical impedance analysis. J Appl Physiol (1985) 2000, 89, 465-471.

20. Phillips, A.; Strobl, R.; Vogt, S.; Ladwig, K.H.; Thorand, B.; Grill, E. Sarcopenia is associated with disability status-results from the KORA-Age study. Osteoporos Int 2017, 28, 2069-2079.

21. CIR Systems Inc. The GAITRite electronic walkway measurements and definitions. Haverton, CIR Systems 2006.

22. German Nutrition Society. New reference values for vitamin D. Ann Nutr Metab 2012, 60, 241-246.

23. Cavalier, E.; Delanaye, P.; Nyssen, L.; Souberbielle, J.C. Problems with the PTH assays. Ann Endocrinol (Paris) 2015, 76, 128-133.

24. Greenland, S.; Pearl, J.; Robins, J.M. Causal diagrams for epidemiologic research. Epidemiology 1999, $10,37-48$.

25. Textor, J.; Hardt, J.; Knuppel, S. DAGitty: a graphical tool for analyzing causal diagrams. Epidemiology 2011, 22, 745.

26. Ahrens, W.; Bellach, B.-M.; Jöckel, K.-H. [Measurement and quantification of sociodemographic characteristics in epidemiological studies]. German Society for Medical Informatics, Biometry and Epidemiology (GMDS). 1998.

27. Keller, H.H.; Goy, R.; Kane, S.L. Validity and reliability of SCREEN II (Seniors in the community: risk evaluation for eating and nutrition, Version II). Eur J Clin Nutr 2005, 59, 1149-1157.

28. Meisinger, C.; Lowel, H.; Thorand, B.; Doring, A. Leisure time physical activity and the risk of type 2 diabetes in men and women from the general population. The MONICA/KORA Augsburg Cohort Study.

Diabetologia 2005, 48, 27-34.

29. Mühlberger, N.; Behrend, C.; Stark, R.; Holle, R. [Database-supported identification and entry of drug data in health studies - experience with the IDOM software]. Informatik Biometrie und Epidemiologie in Medizin und Biologie 2003, 34, 601-611.

30. Schwab, S.; Heier, M.; Schneider, A.; Fischer, B.; Huth, C.; Peters, A.; Thorand, B. The use of dietary supplements among older persons in southern Germany - results from the KORA-age study. J Nutr Health Aging 2014, 18, 510-519.

31. Conzade, R.; Koenig, W.; Heier, M.; Schneider, A.; Grill, E.; Peters, A.; Thorand, B. Prevalence and Predictors of Subclinical Micronutrient Deficiency in German Older Adults: Results from the Population-Based KORA-Age Study. Nutrients 2017, 9.

32. Holle, R.; Hochadel, M.; Reitmeir, P.; Meisinger, C.; Wichmann, H.E. Prolonged recruitment efforts in health surveys: effects on response, costs, and potential bias. Epidemiology 2006, 17, 639-643.

33. Dam, T.T.; von Muhlen, D.; Barrett-Connor, E.L. Sex-specific association of serum vitamin D levels with physical function in older adults. Osteoporos Int 2009, 20, 751-760.

34. Muir, S.W.; Montero-Odasso, M. Effect of vitamin D supplementation on muscle strength, gait and balance in older adults: a systematic review and meta-analysis. J Am Geriatr Soc 2011, 59, 2291-2300.

35. Beaudart, C.; Buckinx, F.; Rabenda, V.; Gillain, S.; Cavalier, E.; Slomian, J.; Petermans, J.; Reginster, J.Y.; Bruyere, O. The effects of vitamin D on skeletal muscle strength, muscle mass, and muscle power: a systematic review and meta-analysis of randomized controlled trials. J Clin Endocrinol Metab 2014, 99, 43364345 .

36. Rosendahl-Riise, H.; Spielau, U.; Ranhoff, A.H.; Gudbrandsen, O.A.; Dierkes, J. Vitamin D supplementation and its influence on muscle strength and mobility in community-dwelling older persons: a systematic review and meta-analysis. J Hum Nutr Diet 2017, 30, 3-15.

37. Vogt, S.; Decke, S.; de Las Heras Gala, T.; Linkohr, B.; Koenig, W.; Ladwig, K.H.; Peters, A.; Thorand, B. Prospective association of vitamin D with frailty status and all-cause mortality in older adults: Results from the KORA-Age Study. Prev Med 2015, 73, 40-46.

38. Kelley, G.A.; Kelley, K.S. Is sarcopenia associated with an increased risk of all-cause mortality and functional disability? Exp Gerontol 2017, 96, 100-103. 
39. Beaudart, C.; Zaaria, M.; Pasleau, F.; Reginster, J.Y.; Bruyere, O. Health Outcomes of Sarcopenia: A Systematic Review and Meta-Analysis. PLoS One 2017, 12, e0169548.

40. de Souza Genaro, P.; de Medeiros Pinheiro, M.; Szejnfeld, V.L.; Martini, L.A. Secondary

hyperparathyroidism and its relationship with sarcopenia in elderly women. Arch Gerontol Geriatr 2015, 60 , 349-353.

41. Kyle, U.G.; Bosaeus, I.; De Lorenzo, A.D.; Deurenberg, P.; Elia, M.; Manuel Gomez, J.; Lilienthal Heitmann, B.; Kent-Smith, L.; Melchior, J.C.; Pirlich, M., et al. Bioelectrical impedance analysis-part II: utilization in clinical practice. Clin Nutr 2004, 23, 1430-1453. 